\title{
Whole-Genome Sequencing Identifies the Egl Nine Homologue 3 (egln3/phd3) and Protein Phosphatase 1 Regulatory Inhibitor Subunit 2 (PPP1R2P1) Associated with High-Altitude Polycythemia in Tibetans at High Altitude
}

\author{
Luobu Gesang, ${ }^{1,2}$ Lamu Gusang, ${ }^{1,2}$ Ciren Dawa,, ${ }^{1,2}$ Gawa Gesang, ${ }^{1,2}$ and Kang Li ${ }^{1}$ \\ ${ }^{1}$ High Altitude Medical Research Institute of Tibet, Lhasa 850000, China \\ ${ }^{2}$ Department of Cardiology, People's Hospital of Tibet Autonomous Region, Lhasa 850000, China \\ Correspondence should be addressed to Kang Li; 807751243@qq.com
}

Received 18 June 2019; Accepted 6 September 2019; Published 7 November 2019

Academic Editor: Hubertus Himmerich

Copyright (C) 2019 Luobu Gesang et al. This is an open access article distributed under the Creative Commons Attribution License, which permits unrestricted use, distribution, and reproduction in any medium, provided the original work is properly cited.

\begin{abstract}
Background. The hypoxic conditions at high altitudes are great threats to survival, causing pressure for adaptation. More and more high-altitude denizens are not adapted with the condition known as high-altitude polycythemia (HAPC) that featured excessive erythrocytosis. As a high-altitude sickness, the etiology of HAPC is still unclear. Methods. In this study, we reported the wholegenome sequencing-based study of 10 native Tibetans with HAPC and 10 control subjects followed by genotyping of selected 21 variants from discovered single nucleotide variants (SNVs) in an independent cohort (232 cases and 266 controls). Results. We discovered the egl nine homologue 3 (egln3/phd3) (14q13.1, rs1346902, $P=1.91 \times 10^{-5}$ ) and PPP1R2P1 (Protein Phosphatase 1 Regulatory Inhibitor Subunit 2) gene $(6 \mathrm{p} 21.32$, rs521539, $P=0.012)$. Our results indicated an unbiased framework to identify etiological mechanisms of HAPC and showed that egln3/phd3 and PPP1R2P1 may be associated with the susceptibility to HAPC. Egln3/phd3b is associated with hypoxia-inducible factor subunit $\alpha$ (HIF $\alpha$ ). Protein Phosphatase 1 Regulatory Inhibitor is associated with reactive oxygen species (ROS) and oxidative stress. Conclusions. Our genome sequencing conducted in Tibetan HAPC patients identified egln3/phd3 and PPP1R2P1 associated with HAPC.
\end{abstract}

\section{Introduction}

In excess of 140 million humans have primarily lived in the high-altitude regions of various locations around the world, including the Qinghai-Tibet plateau in Asia, the Andes Mountains of South America, and the Ethiopian plateau in East Africa [1]. All dwellers in plateaus like native Tibetans of the Qinghai-Qinghai-Tibet plateau are regarded as one of the best dwellers adapted to high altitudes. They possess heritable adaptations to the hypoxic condition [2], as they display lower hemoglobin and hematocrit [3], higher oxygen saturation of blood [4], and better work efficiency [5], which are conducive to adapting the high-altitude and hypoxic situations. However, some Tibetans living at high altitude with genetic adaptations remain maladapted and possess symptoms of high-altitude polycythemia (HAPC).
HAPC is diagnosed with excessive erythrocytosis encountered by around $10 \%$ of the population residing at the Qinghai-Tibet plateau [6, 7]. HAPC shows several impacts on blood viscosity with various harmful consequences such as dysfunction of microcirculation, vascular thrombosis, extensive organ damage, sleep disturbances, and even death $[7,8]$. In high-altitude dwellers, hypobaric hypoxia is thought to be one of the primary initiators of HAPC. However, the mechanisms underlying the pathogenesis and genetic basis of HAPC have not been well understood.

In recent years, the genetic basis underlying adaptation to high altitude in Tibetans has been elucidated by using exome and single nucleotide polymorphism (SNP) array data [9-11]. Furthermore, gene candidate approaches in Tibetan have identified that genomic variants in EPAS1, CYP17A1, CYP2E1, and mitochondrial DNA 10609T are associated with HAPC 
TABLE 1: Clinical characterization between two groups.

\begin{tabular}{|c|c|c|c|c|c|c|c|c|}
\hline Group & Sample & Gender & Age & Living place & Living altitude & Systolic blood pressure & Diastolic blood pressure & $\mathrm{Hb}$ \\
\hline \multirow{10}{*}{ HAPC } & D1WGC025333 & $\mathrm{F}$ & 56 & Lhasa & $3650 \mathrm{~m}$ & 120 & 80 & 235 \\
\hline & D2WGC025334 & M & 46 & Xigaze & $3900 \mathrm{~m}$ & 120 & 80 & 244 \\
\hline & D3WGC025335 & M & 62 & Lhasa & $3650 \mathrm{~m}$ & 120 & 80 & 220 \\
\hline & D4WGC025336 & M & 59 & $\mathrm{Naqu}$ & $4500 \mathrm{~m}$ & 120 & 80 & 274 \\
\hline & D5WGC025337 & M & 37 & Xigaze & $3900 \mathrm{~m}$ & 130 & 110 & 230 \\
\hline & D6WGC025338 & M & 30 & Xigaze & $3900 \mathrm{~m}$ & 120 & 70 & 219 \\
\hline & D8WGC025340 & M & 34 & Shannan & $3500 \mathrm{~m}$ & 100 & 60 & 215 \\
\hline & D10WGC025341 & M & 48 & Shannan & $3500 \mathrm{~m}$ & 124 & 80 & 249 \\
\hline & D13WGC025344 & M & 36 & Shannan & $3500 \mathrm{~m}$ & 116 & 70 & 237 \\
\hline & D15WGC025346 & M & 46 & Linzhi & $3000 \mathrm{~m}$ & 100 & 60 & 240 \\
\hline \multirow{10}{*}{ Control } & Z1WGC025347 & $\mathrm{F}$ & 60 & Xigaze & $3900 \mathrm{~m}$ & 110 & 70 & 140 \\
\hline & Z2WGC025348 & M & 64 & Xigaze & $3900 \mathrm{~m}$ & 120 & 80 & 146 \\
\hline & Z3WGC025349 & $\mathrm{F}$ & 50 & Lhasa & $3650 \mathrm{~m}$ & 120 & 80 & 163 \\
\hline & Z4WGC025350 & M & 52 & Xigaze & $3900 \mathrm{~m}$ & 120 & 80 & 162 \\
\hline & Z5WGC025351 & M & 63 & Shannan & $3500 \mathrm{~m}$ & 120 & 80 & 162 \\
\hline & Z6WGC025352 & $\mathrm{F}$ & 42 & Xigaze & $3900 \mathrm{~m}$ & 116 & 76 & 146 \\
\hline & Z7WGC025353 & M & 41 & Xigaze & $3900 \mathrm{~m}$ & 130 & 90 & 163 \\
\hline & Z8WGC025354 & M & 48 & Shannan & $3500 \mathrm{~m}$ & 110 & 82 & 162 \\
\hline & Z13WGC025359 & M & 44 & Shannan & $3500 \mathrm{~m}$ & 102 & 56 & 174 \\
\hline & Z14WGC025360 & $\mathrm{F}$ & 65 & Xigaze & $3900 \mathrm{~m}$ & 120 & 80 & 170 \\
\hline
\end{tabular}

Note: HAPC: high-altitude polycythemia; M: male; F: female.

[12-15]. However, comprehensive genome-wide scanning to detect the variations associated with HAPC has not been performed. It has been known that the variants that occurred in the genome-wide association study (GWAS) may account for some diseases with only relatively modest proportions and numerous deleterious variants with low frequency in the population.

With the technical development of high-throughput sequencing, it provides an opportunity to resequence multiple genetic regions and recognize novel risk alleles in diseases including high-altitude sickness [1, 16-18]. To dissect the genetic mechanisms underlying HAPC, we compared genetic variations between native Tibetans with HAPC and adapted subjects without HAPC by whole-genome sequencing (WGS) of 20 individuals, and selected variants were further genotyped in an additional larger case-control cohort. Our investigation revealed the egl nine homologue 3 (egln3/phd3) (14q13.1) and PPP1R2P1 (Protein Phosphatase 1 Regulatory Inhibitor Subunit 2) gene (6p21.32) associated with HAPC.

\section{Methods}

2.1. Patients and Healthy Control Subjects. As the same ethnicity, HAPC was collected in healthy control subjects and matched with native high-altitude Tibetans. In this study, 10 patients with HAPC and 10 control subjects without HAPC that were performed in Tibet area (Table 1) were recruited. Based on the International Consensus Statement [7], (1) HAPC was diagnosed with an $\mathrm{Hb}$ concentration of at least $19 \mathrm{~g} / \mathrm{dL}$ and $21 \mathrm{~g} / \mathrm{dL}$ for women and men, respectively, and (2) HAPC patients were local Tibetans normally living at 3600 to $4500 \mathrm{~m}$. Blood samples for WGS were collected, and each subject was informed and gave written consent.

2.2. Whole-Genome Sequencing. Blood DNA was isolated from each subject, and the desired length fragments were gel purified. According to the manufacturer's instruction, DNA cluster preparation and adaptor ligation were performed with the library preparation kit (Illumina). WGS was performed on Illumina HiseqX ten Platform with 150 base pairs (bps) paired-end reads.

2.3. Read Alignment and Variant Calling. All reads were mapped to human reference genome by BWA [19] with default parameter. For downstream filtering, picard tools (http://broadinstitute.github. io/picard/) were used to mark PCR duplicates. We used Genome Analysis Toolkit (GATK) to adjust the alignments via GATK indel realignment and score recalibration modules [20]. We finally called and filtered SNVs using the GATK Unified Genotyper under default parameters. With the Ensembl annotated database, the resulting SNVs were annotated by ANNOVAR [21] according to the locations and expected effect on encoded gene products. According to dnSNP, SNVs were identified into "newly identified" and "known" variants.

2.4. SNP Discovery Stage. The allele frequency distribution of 10 cases and 10 controls and variants with significant difference $(P<0.01)$ were analyzed by the Fisher exact test in allele frequency. To select the SNVs for association mapping, the pathway enrichment analysis using WebGestalt 
TABLE 2: Summary of whole-genome sequencing and variant call statistics per individual.

\begin{tabular}{|c|c|c|c|c|c|c|c|c|}
\hline Category & Sample & $\begin{array}{l}\text { Paired-end reads } \\
\quad \text { (million) }\end{array}$ & Mapped rate & Mean depth & $\begin{array}{l}\text { Coverage of } \\
\text { genome }\end{array}$ & Final SNVs & SNVs (\%) & Nonsilent SNVs \\
\hline \multirow{10}{*}{ HAPC } & D1WGC025333 & 421.0 & $99.6 \%$ & 42.5 & $99.7 \%$ & $2,672,708$ & 94.2 & 6,600 \\
\hline & D2WGC025334 & 417.6 & $99.5 \%$ & 41.8 & $99.8 \%$ & $3,506,627$ & 94.1 & 9,610 \\
\hline & D3WGC025335 & 404.4 & $99.7 \%$ & 41.1 & $99.8 \%$ & $3,536,613$ & 93.5 & 9,926 \\
\hline & D4WGC025336 & 408.8 & $99.5 \%$ & 41.3 & $99.7 \%$ & $3,532,401$ & 93.7 & 9,918 \\
\hline & D5WGC025337 & 410.3 & $99.6 \%$ & 41.6 & $99.8 \%$ & $3,511,589$ & 94.2 & 9,605 \\
\hline & D6WGC025338 & 402.3 & $99.5 \%$ & 40.7 & $99.7 \%$ & $3,507,517$ & 94.1 & 9,595 \\
\hline & D8WGC025340 & 366.2 & $40.7 \%$ & 14.4 & $99.5 \%$ & $3,362,350$ & 93.2 & 9,593 \\
\hline & D10WGC025341 & 412.0 & $97.4 \%$ & 40.7 & $99.8 \%$ & $3,529,927$ & 93.6 & 9,760 \\
\hline & D13WGC025344 & 392.8 & $99.5 \%$ & 39.3 & $99.8 \%$ & $3,538,731$ & 93.5 & 9,860 \\
\hline & D15WGC025346 & 401.3 & $99.4 \%$ & 40.3 & $99.7 \%$ & $3,510,739$ & 93.8 & 9,687 \\
\hline \multirow{10}{*}{ Control } & Z1WGC025347 & 429.5 & $99.5 \%$ & 43.5 & $99.1 \%$ & $3,546,349$ & 93.5 & 9,750 \\
\hline & Z2WGC025348 & 365.3 & $99.8 \%$ & 37 & $99.7 \%$ & $3,529,029$ & 93.4 & 9,835 \\
\hline & Z3WGC025349 & 305.2 & $99.6 \%$ & 30.9 & $99.0 \%$ & $3,561,607$ & 93.3 & 9,940 \\
\hline & Z4WGC025350 & 429.0 & $99.6 \%$ & 43.4 & $99.7 \%$ & $3,511,388$ & 93.7 & 9,629 \\
\hline & Z5WGC025351 & 422.8 & $99.6 \%$ & 42.6 & $99.7 \%$ & $3,513,151$ & 93.8 & 9,467 \\
\hline & Z6WGC025352 & 363.9 & $99.8 \%$ & 37 & $99.1 \%$ & $3,544,574$ & 93.6 & 9,735 \\
\hline & Z7WGC025353 & 412.8 & $99.5 \%$ & 41.8 & $99.8 \%$ & $3,535,839$ & 93.6 & 9,857 \\
\hline & Z8WGC025354 & 420.8 & $99.4 \%$ & 42.7 & $99.7 \%$ & $3,544,492$ & 93.6 & 9,705 \\
\hline & Z13WGC025359 & 413.5 & $99.5 \%$ & 41.9 & $99.8 \%$ & $3,534,525$ & 93.5 & 9,841 \\
\hline & Z14WGC025360 & 412.1 & $99.5 \%$ & 41.9 & $99.1 \%$ & $3,561,399$ & 93.4 & 9,821 \\
\hline
\end{tabular}

(http://bioinfo.vanderbilt.edu/webgestalt/) was performed by the distribution of genes with different allele frequencies. We used the hypergeometric test and Benjamini-Hochberg FDR to determine the significance of enrichment and adjust the multiple testing, respectively. This analysis revealed pathways including the HIF-1 signaling pathway and hematopoietic cell lineage [22] were associated with high-attitude adaption. We selected $21 \mathrm{SNV}$ s in the two pathways from SNVs with different allele frequencies for genotyping in the stage of the association study.

2.5. Genotyping and Statistical Analysis. Genotyping of the selected 21 variants was performed with Sequenom MassARRAY or Snapshot system in an additional 232 HAPC cases and 266 controls. The primer of genotyping was designed by use of Primer 5.0. The frequency of alleles was calculated by genotype frequency in HAPC patients and control subjects, and the intergroup differences were counted using the Fisher exact test. $P$ value for multiple testing was adjusted by Benjamini-Hochberg FDR. QQ plot was produced using the man statistics package of $\mathrm{R}$ (http://cran.r-project.org/ web/packages/qqman/). We use the chi-square test to assess deviations from Hardy-Weinberg equilibrium.

\section{Results}

3.1. Whole-Genome Sequencing and Single Nucleotide Variants $(S N V s)$. After extracting DNA from the peripheral blood, the whole-genome sequencing of 10 native Tibetans with HAPC and 10 adapted subjects without HAPC was performed by using next-generation sequencing technology (Supplementary Table S1). As a result, there were more than 360 million pairedend reads for each subject (Table 2). More than $99.7 \%$ of the reference genome was covered, and we sequenced the genome with a mean depth of $39 x$ per individual. After applying stringent quality controls, we obtained $69,591,555$ SNVs (single nucleotide variants), of which 191,734 (0.28\%) were nonsilent mutations (nonsynonymous and splice site mutations). Comparing with 1000 Genome Project [23] gave $94 \%$ of SNVs known in 1000 Genomes Phase 1 ASN.

To discover the susceptibility loci associated with HAPC, two-stage studies including SNP discovery study and replication study were performed. To select the candidate SNVs for association mapping, we applied the Fisher exact test to WGS data and identified 33,662 SNVs $(P<$ 0.01 ) in allele frequency between native Tibetans with HAPC and adapted subjects without HAPC, of which 72 were nonsilent SNVs (Supplementary Table S2). Pathway enrichment analysis was performed on the genes with significant difference in allele frequency and revealed pathways including the HIF-1 signaling pathway [24] and hematopoietic cell lineage [22] that were associated with high-attitude adaption (Supplementary Table S3). To explore the association between HAPC and the two pathways, we subjected 21 SNVs that are in the two pathways and also showed allele frequency difference in the above Fisher exact test for genotyping in the stage of the association study (Supplementary Table S4).

3.2. Association Study. Associations between the 21 selected variants and HAPC were evaluated by genotyping in an 


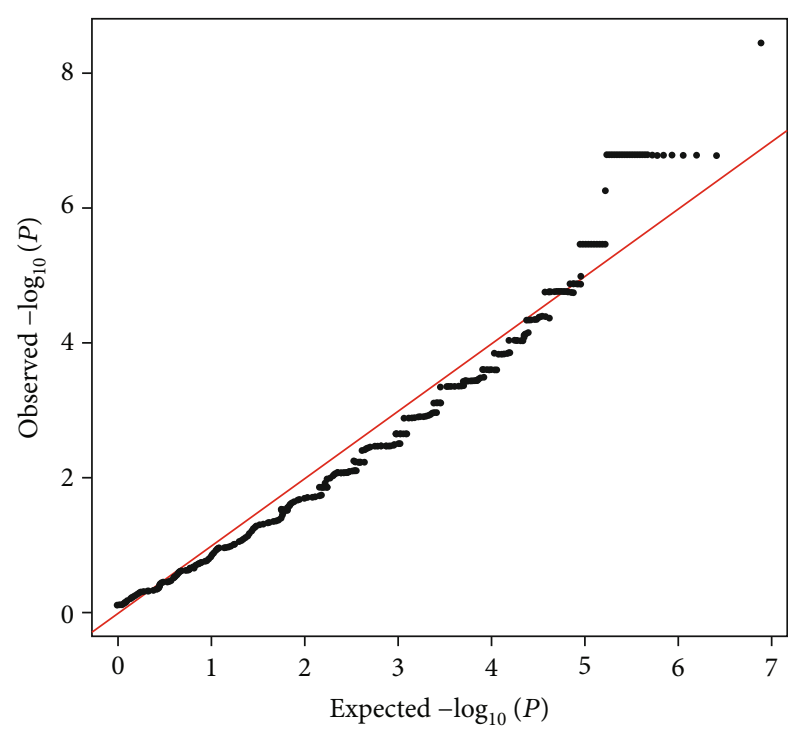

Figure 1: QQ plot indicates the differences between observed and expected $-\log _{10}(P$ value $)$.

additional 232 Tibetans with HAPC and 266 control subjects (Supplementary Table S5). Most of the observed associations showed close to the expected distribution based on the quantile-quantile (QQ) plot, which is consistent with the null hypothesis of no association (Figure 1). Two loci exceeded significance in association with HAPC (Table 3): egln3/phd3 at rs1346902 in EGLN3 on $14 \mathrm{q} 13.1(P=1.91 \times$ $10^{-5}$, odds ratio $(\mathrm{OR})=0.57,95 \%$ confidence intervals $(\mathrm{CI})$ : 0.44-0.74) and PPP1R2P1 at rs521539 in the region between HLA-DRB1 and HLA-DQA1 on 6p21.32 $(P=0.012, \mathrm{OR}=$ 1.41, 95\% CI: 1.08-1.84). The allele frequencies of other variants between these two groups were not significantly different. Furthermore, the overlap of these two associated SNPs was evaluated with the Encyclopedia of DNA Elementsannotated genomic elements (Supplementary Table S6). There were histone modifications at promoter or enhancer; DNase hypersensitivity sites, binding proteins, and motifs changed in both SNVs.

3.3. The Analysis of Clinical and Genetic Correlation. We next measured whether tegln3/phd3 and PPP1R2P1 were concerned with clinical characters between the HAPC group and the control group using analysis of variance method (Figure 1). In all Tibetan subjects, we found the obvious correlation $(P<0.01)$ between tegln $3 / \mathrm{phd} 3$ and hemoglobin ( $\mathrm{HGB}$ or $\mathrm{Hb} ; F$ value $=14.1, P=0.0002$ ), red blood cell count $(\mathrm{RBC} ; F$ value $=12.2, P=0.0005)$, hematocrit $(\mathrm{HCT} ; F$ value $=9.2, P=0.003$ ), and direct bilirubin (DBIL; $F$ value $=8.6, P=0.004)$. Tibetan subjects with CC genotype showed lower $\mathrm{Hb}$ than those with either the CG $\left(P=1.8 \times 10^{-5}\right)$ or the GG $\left(P=5 \times 10^{-5}\right)$ genotype (Figure 2(a)). Similarly, the lower HGB, HCT, and DBIL were also observed in individuals with $\mathrm{CC}$ genotype compared with CG or GG individuals (Figures 2(b)-2(d)). Additionally, we also found a significant association between PPP1R2P1 $(\mathrm{A} / \mathrm{G})$ and the albumin to globulin ratio (Figure 2(e); $F$ value $=6.8, P=0.009)$.

\section{Discussion}

In the present study, we used whole-genome sequencing of native Tibetans with HAPC and adapted subjects without HAPC to identify alleles' associated risk in previously unidentified HAPC susceptibility genes. We selected 21 variants from our WGS data for further evaluation in an additional larger cohort of Tibetans with HAPC and controls. The differences in the SNPs of tegln3/phd3 (C/G) and PPP1R2P1 (A/G) between the two groups are found for the first time in this present paper.

SNP rs1346902 is located in the intron region (transcript ID: ENST00000487915 and ENST00000551935) of EGLN3 (also known as PHD3) that belongs to the Egl-9 (EGLN) family. EGLN3, along its paralogous genes EGLN1 and EGLN2, can catalyze the hydroxylation of hypoxiainducible factor subunit $\alpha(\mathrm{HIF} \alpha)$, leading to increased ubiquitination and proteasomal degradation of $\mathrm{HIF} \alpha$ and decreased HIF $\alpha$ activity [25]. Upregulated EGLN3 is related to apoptosis in sympathetic neurons [26], p53-induced growth arrest in RAS-transformed embryo fibroblasts [27], and differentiation of $\mathrm{C} 2 \mathrm{C} 12$ myoblasts [28]. EGLN3 also plays important roles in human endothelial cells [29] and cancers, including pancreatic cancer [30] and glioblastoma [31]. Previous study also identifies that EGLN3 can negatively regulate the $\mathrm{NF}-\kappa \mathrm{B}$ pathway, which is a crucial regulator of cell differentiation, inflammation, stress responses, and immunity [32]. Although association analysis with microsatellite markers in an Andean high-altitude population shows a possible association between EGLN3 (marker D14S1049) and severe polycythemia [33], no susceptible association between SNPs in EGLN3 and HAPC is reported. Here, we found a susceptibility locus in EGLN3 for HAPC. We also found significantly lower $\mathrm{Hb}$ and $\mathrm{RBC}$ in the subjects with rs1346902 C/C allele (Figures 2(a) and 2(b)), supporting and extending the potential role of EGLN3 in HAPC. Therefore, the significant difference of genotypes between two groups for SNP rs1346902 (C/C) was the beneficial allele of HAPC and it might contribute to high-altitude adaption in Tibetans with lower $\mathrm{Hb}$ and $\mathrm{RBC}$.

A large number of genomic surveys about nucleotide polymorphism among humans in high-altitude environments have offered evidence for genes in the HIF oxygen signaling pathway, among which EPAS1 and EGLN1 are notable [9, 10, 34-36]. EPAS1, EGLN1, VEGFA, CYP17A1, and $C Y P 2 E 1$ in the HIF pathway are also shown to be associated with high-altitude sickness, including HAPC [12-14, 37-39]. The finding of susceptibility SNP in EGLN3 for HAPC in our study further supported the key role of the HIF oxygen signaling pathway in high-altitude adaption and relevant diseases.

Human leukocyte antigen (HLA) loci have been associated with more than 100 diseases [40]. HLA-DRB1 and $H L A-D Q A 1$ play an important role in the immune system, which belong to the classical HLA class II molecules. They can take part in recognizing and presenting antigens to $\mathrm{T}$ cells and are mainly expressed in B cells, dendritic cells, and macrophages. In addition, several studies have found a connection between HLA class II molecules and immune- 
TABLE 3: Association between HAPC and 2 variants.

\begin{tabular}{lccccccc}
\hline $\begin{array}{l}\text { MAF } \\
\text { Chr. }\end{array}$ & Near gene & Variants & Allele & Case & Control & $P$ value & OR (95\% CI) \\
\hline $14 q 13.1$ & EGLN3 & rs1346902 & C/G & 0.342 & 0.477 & 0.0000191 & $0.57(0.44-0.74)$ \\
$6 \mathrm{p} 21.32$ & HLA-DRB1-HLA-DQA1 & rs521539 & A/G & 0.561 & 0.475 & 0.012 & $1.41(1.08-1.84)^{*}$ \\
\hline
\end{tabular}

Chr.: chromosome; MAF: minor allele frequency; CI: confidence interval. Alleles are shown as minor allele/major allele. * Odds ratio and CI for A allele vs. G allele. The egl nine homologue 3 (egln3/phd3) (14q13.1, rs1346902, $P=1.91 \times 10^{-5}$ ) and PPP1R2P1 (Protein Phosphatase 1 Regulatory Inhibitor Subunit 2) gene (6p21.32, rs521539, $P=0.012)$.

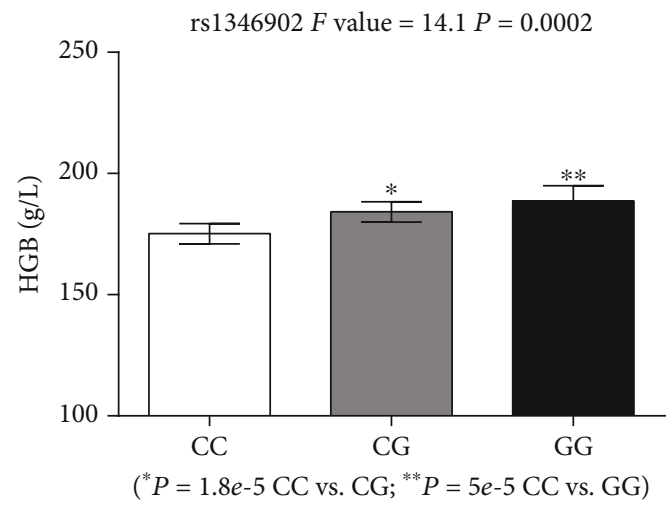

(a)

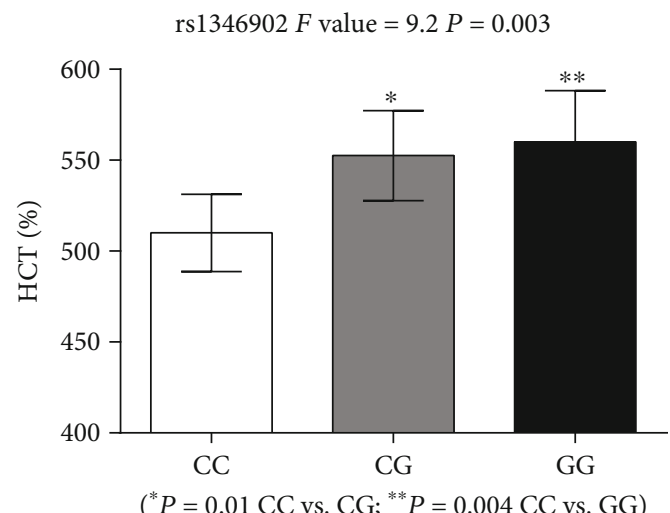

(c)

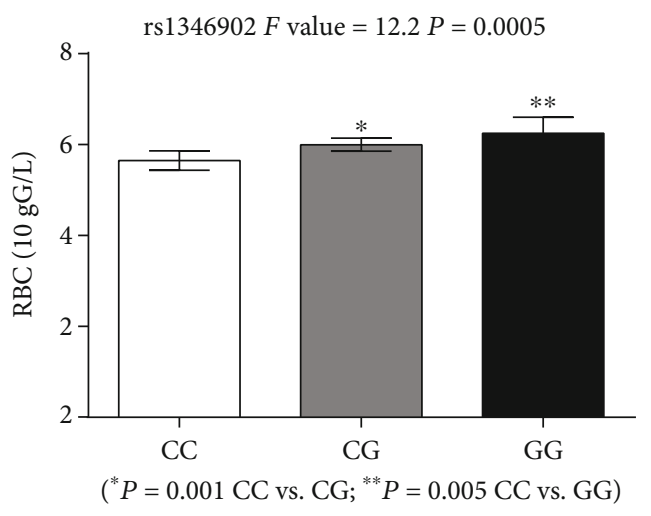

(b)

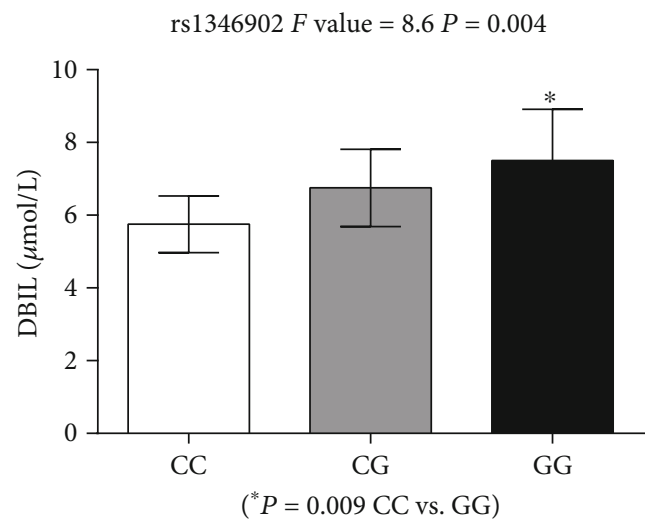

(d)

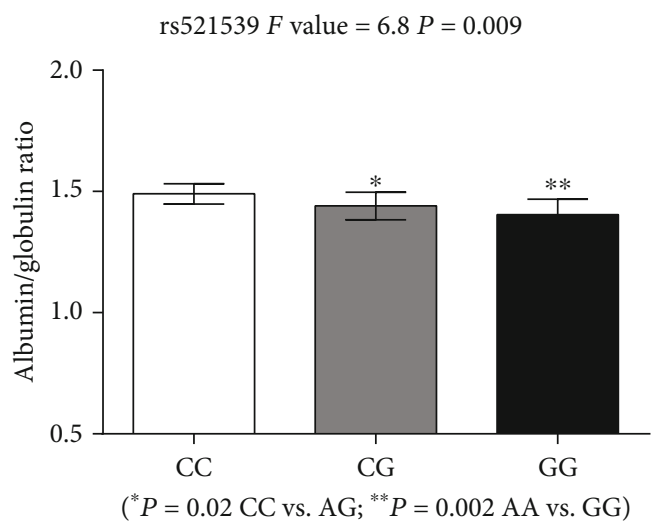

(e)

Figure 2: Clinical and genetic association analysis for the egl nine homologue $3\left(\right.$ egln3/phd3) $\left(14 \mathrm{q} 13.1, \mathrm{rs} 1346902, P=1.91 \times 10^{-5}\right)$ and PPP1R2P1 (Protein Phosphatase 1 Regulatory Inhibitor Subunit 2) gene $(6 \mathrm{p} 21.32$, rs521539, $P=0.012)$. Significant correlation was found between AX9 and NKX2-1 and hemoglobin (HGB or Hb) (a), red blood cell count (RBC) (b), hematocrit (HCT) (c), and direct bilirubin (DBIL) (d). PPP1R2P1 was also significantly correlated with the albumin to globulin ratio (e). The association analysis was examined by analysis of variance method, and differences between genotypes were evaluated with a $t$-test. 
mediated disorders, such as susceptibility loci in HLA-DRB1 for sarcoidosis [41] and pemphigus [42] and susceptibility loci in HLA-DQA1 for achalasia [43] and celiac diseases [44].

In our study, we also found that there were significant differences $(P=0.012)$ in the allele frequency of PPP1R2P1 near $H L A-D R B 1 / D Q A 1$ between the HAPC group and the control group, with high-frequency A allele among HAPC (57\%) than controls (47\%). Our finding was consistent with positive selection of HLA loci (HLA-DRA, HLA$D Q B 1 / H L A-D Q A 2)$ in Ethiopian population [45] and significantly associated with the high-altitude pulmonary edema of HLA-DR6 and HLA-DQ4 [46]. Our results also revealed a significant correlation between PPP1R2P1 (A/G) and albu$\mathrm{min} /$ globulin ratio (Figure 2(e)), which is an indicator of liver and kidney disorders. The implication of HLA suggests a role of inflammation perhaps associated with the kidneys or livers. Previous studies show that chronic hypoxia induced hypertension and erythrocytosis leads to renal injury and inflammation in rats [47]. The similar effect between HLA and HAPC may exist in humans. Protein Phosphatase 1 Regulatory Inhibitor has been reported to be associated with reactive oxygen species (ROS) and oxidative stress [48].

The primary limitation of our research was the small case-control cohort in the discovery phase, which might result in low power of detection of candidate susceptibility loci. Moreover, the functional role of newly identified susceptibility loci was needed to be verified by some experiments. We will pay more attention to the expression pattern of these genes and related regulatory pathways in HAPC patients.

\section{Conclusions}

In summary, our genome sequencing conducted in Tibetan HAPC patients identified two new susceptibility loci at $14 \mathrm{q} 13.1$ and $6 \mathrm{p} 21.32$ and offered to our knowledge of the genetic architecture of HAPC. Further study about these two loci was likely to improve our knowledge on the etiology of HAPC.

\section{Abbreviations}

HAPC: High-altitude polycythemia

SNP: $\quad$ Single nucleotide polymorphism

GWAS: Genome-wide association study

WGS: Whole-genome sequencing

SNVs: Single nucleotide variants

$\operatorname{HIF} \alpha$ : Hypoxia-inducible factor subunit $\alpha$

HLA: Human leukocyte antigen.

\section{Data Availability}

Our datasets are presented within the manuscript and additional supporting files.

\section{Ethical Approval}

The experimental protocol was established according to the ethical guidelines of the Helsinki Declaration and approved by the ethics committee of People's Hospital of Tibet Autono- mous Region, Lhasa, China. Besides, all experiments were performed in accordance with relevant guidelines and regulations

\section{Consent}

Written informed consent was obtained from the individual participants.

\section{Conflicts of Interest}

The authors declare no actual or perceived competing interests.

\section{Authors' Contributions}

L.G., K.L., L.G., K.L., C.D., Y.B., R.T., and M.N. provided materials and performed sample preparation. Q.D. and G.G. performed laboratory work. L.G., K.L., and A san performed high-throughput genome sequencing. L.G., K.L., and A san analyzed genomic sequence and SNP data. L.G. and K.L. wrote the manuscript with contributions from all coauthors. All authors read and approved the final manuscript.

\section{Acknowledgments}

This study was supported by a grant from the National "Twelfth Five-Year" Plan for Science \& Technology Support of China (2013BAI05B04), the Key project for Natural Science Foundation in Tibet Autonomous Region, China (2012), and the National Natural Science Foundation of China (NSFC) (No. 81960029).

\section{Supplementary Materials}

Supplementary Table S1: sequence data for HAPC and control subjects. Supplementary Table S2: list of 72 nonsilent SNPs with $P<0.01$ in allele frequency between cases and healthy controls in the discovery phase. Supplementary Table S3: pathway enrichment analysis on the genes with significant difference in allele frequency between 10 cases and 10 controls. Supplementary Table S4: the selected 21 SNPs for genotyping. Supplementary Table S5: characteristics of case and control subjects in the genotyping phase. Supplementary Table S6: functional annotation of SNPs correlated with rs1346902 and rs521539 $\left(r^{2}>0.8\right)$ using data from ENCODE. (Supplementary Materials)

\section{References}

[1] D. Zhou, N. Udpa, R. Ronen et al., "Whole-genome sequencing uncovers the genetic basis of chronic mountain sickness in Andean highlanders," American Journal of Human Genetics, vol. 93, no. 3, pp. 452-462, 2013.

[2] C. Jeong and A. Di Rienzo, "Adaptations to local environments in modern human populations," Current Opinion in Genetics \& Development, vol. 29, pp. 1-8, 2014.

[3] T. Y. Wu, "Chronic mountain sickness on the QinghaiQinghai-Tibet plateau," Chinese Medical Journal, vol. 118, no. 2, pp. 161-168, 2005. 
[4] S. Niermeyer, P. Yang, D. Shanmina, J. Zhuang, and L. G. Moore, "Arterial oxygen saturation in Tibetan and Han infants born in Lhasa, Tibet," The New England Journal of Medicine, vol. 333, no. 19, pp. 1248-1252, 1995.

[5] J. Zhuang, T. Droma, J. R. Sutton et al., "Smaller alveolar-arterial $\mathrm{O}_{2}$ gradients in Tibetan than Han residents of Lhasa (3658 m)," Respiration Physiology, vol. 103, no. 1, pp. 75-82, 1996.

[6] J. S. Windsor and G. W. Rodway, "Heights and haematology: the story of haemoglobin at altitude," Postgraduate Medical Journal, vol. 83, no. 977, pp. 148-151, 2007.

[7] F. León-Velarde, M. Maggiorini, J. T. Reeves et al., "Consensus statement on chronic and subacute high altitude diseases," High Altitude Medicine \& Biology, vol. 6, no. 2, pp. 147-157, 2005.

[8] W. Guan, Q. Ga, R. Li et al., "Sleep disturbances in long-term immigrants with chronic mountain sickness: a comparison with healthy immigrants at high altitude," Respiratory Physiology \& Neurobiology, vol. 206, pp. 4-10, 2015.

[9] X. Yi, Y. Liang, E. Huerta-Sanchez et al., "Sequencing of 50 human exomes reveals adaptation to high altitude," Science, vol. 329, no. 5987, pp. 75-78, 2010.

[10] T. S. Simonson, Y. Yang, C. D. Huff et al., "Genetic evidence for high-altitude adaptation in Tibet," Science, vol. 329, no. 5987 , pp. 72-75, 2010.

[11] E. Huerta-Sánchez, X. Jin, Z. B. Asan et al., "Altitude adaptation in Tibetans caused by introgression of Denisovan-like DNA," Nature, vol. 512, no. 7513, pp. 194-197, 2014.

[12] Y. Chen, C. Jiang, Y. Luo, F. Liu, and Y. Gao, “An EPAS1 haplotype is associated with high altitude polycythemia in male Han Chinese at the Qinghai-Qinghai-Tibet plateau," Wilderness \& Environmental Medicine, vol. 25, no. 4, pp. 392-400, 2014.

[13] J. Xu, Y. Z. Yang, F. Tang et al., "CYP17A1 and CYP2E1 variants associated with high altitude polycythemia in Tibetans at the Qinghai-Tibet plateau," Gene, vol. 566, no. 2, pp. 257-263, 2015.

[14] J. Xu, Y. Z. Yang, F. Tang, Q. Ga, W. Tana, and R. L. Ge, "EPAS1 gene polymorphisms are associated with high altitude polycythemia in Tibetans at the Qinghai-Tibet plateau," Wilderness \& Environmental Medicine, vol. 26, no. 3, pp. 288294, 2015.

[15] C. Jiang, J. Cui, F. Liu et al., "Mitochondrial DNA 10609T promotes hypoxia-induced increase of intracellular ROS and is a risk factor of high altitude polycythemia," PLoS One, vol. 9, no. 1, article e87775, 2014.

[16] H. Tang, X. Jin, Y. Li et al., "A large-scale screen for coding variants predisposing to psoriasis," Nature Genetics, vol. 46, no. 1, pp. 45-50, 2014.

[17] J. I. Kiiski, L. M. Pelttari, S. Khan et al., "Exome sequencing identifies FANCM as a susceptibility gene for triple-negative breast cancer," Proceedings of the National Academy of Sciences of the United States of America, vol. 111, no. 42, pp. 15172-15177, 2014.

[18] CONVERGE consortium, "Sparse whole-genome sequencing identifies two loci for major depressive disorder," Nature, vol. 523, no. 7562, pp. 588-591, 2015.

[19] H. Li and R. Durbin, "Fast and accurate short read alignment with Burrows-Wheeler transform," Bioinformatics, vol. 25, no. 14, pp. 1754-1760, 2009.

[20] A. McKenna, M. Hanna, E. Banks et al., "The Genome Analysis Toolkit: a MapReduce framework for analyzing next-generation
DNA sequencing data," Genome Research, vol. 20, no. 9, pp. 1297-1303, 2010.

[21] K. Wang, M. Li, and H. Hakonarson, “ANNOVAR: functional annotation of genetic variants from high-throughput sequencing data," Nucleic Acids Research, vol. 38, no. 16, article e164, 2010.

[22] P. Li, J. Huang, H. J. Tian, Q. Y. Huang, C. H. Jiang, and Y. Q. Gao, "Regulation of bone marrow hematopoietic stem cell is involved in high-altitude erythrocytosis," Experimental Hematology, vol. 39, no. 1, pp. 37-46, 2011.

[23] Genomes Project C, G. R. Abecasis, A. Auton et al., "An integrated map of genetic variation from 1,092 human genomes," Nature, vol. 491, no. 7422, pp. 56-65, 2012.

[24] A. W. Bigham and F. S. Lee, "Human high-altitude adaptation: forward genetics meets the HIF pathway," Genes \& Development, vol. 28, no. 20, pp. 2189-2204, 2014.

[25] W. G. Kaelin Jr. and P. J. Ratcliffe, "Oxygen sensing by metazoans: the central role of the HIF hydroxylase pathway," Molecular Cell, vol. 30, no. 4, pp. 393-402, 2008.

[26] S. L. Madden, E. A. Galella, D. Riley, A. H. Bertelsen, and G. A. Beaudry, "Induction of cell growth regulatory genes by p53," Cancer Research, vol. 56, no. 23, pp. 5384-5390, 1996.

[27] E. A. Lipscomb, P. D. Sarmiere, R. J. Crowder, and R. S. Freeman, "Expression of the SM-20 gene promotes death in nerve growth factor-dependent sympathetic neurons," Journal of Neurochemistry, vol. 73, no. 1, pp. 429-432, 1999.

[28] J. Fu, K. Menzies, R. S. Freeman, and M. B. Taubman, "EGLN3 prolyl hydroxylase regulates skeletal muscle differentiation and myogenin protein stability," The Journal of Biological Chemistry, vol. 282, no. 17, pp. 12410-12418, 2007.

[29] S. A. Gerber, B. Yatsula, C. L. Maier, T. J. Sadler, L. W. Whittaker, and J. S. Pober, "Interferon-gamma induces prolyl hydroxylase (PHD) 3 through a STAT1-dependent mechanism in human endothelial cells," Arteriosclerosis, Thrombosis, and Vascular Biology, vol. 29, no. 9, pp. 1363-1369, 2009.

[30] Y. Su, M. Loos, N. Giese et al., "PHD3 regulates differentiation, tumour growth and angiogenesis in pancreatic cancer," British Journal of Cancer, vol. 103, no. 10, pp. 1571-1579, 2010.

[31] A. T. Henze, J. Riedel, T. Diem et al., "Prolyl hydroxylases 2 and 3 act in gliomas as protective negative feedback regulators of hypoxia-inducible factors," Cancer Research, vol. 70, no. 1, pp. 357-366, 2010.

[32] J. Fu and M. B. Taubman, "EGLN3 inhibition of NF- B is mediated by prolyl hydroxylase-independent inhibition of I B Kinase Ubiquitination," Molecular and Cellular Biology, vol. 33, no. 15, pp. 3050-3061, 2013.

[33] O. M. Mejia, J. T. Prchal, F. Leon-Velarde, A. Hurtado, and D. W. Stockton, "Genetic association analysis of chronic mountain sickness in an Andean high-altitude population," Haematologica, vol. 90, no. 1, pp. 13-19, 2005.

[34] A. Bigham, M. Bauchet, D. Pinto et al., "Identifying signatures of natural selection in Tibetan and Andean populations using dense genome scan data," PLoS Genetics, D. J. Begun, Ed., vol. 6, no. 9, article e1001116, 2010.

[35] Y. Peng, Z. Yang, H. Zhang et al., "Genetic variations in Tibetan populations and high-altitude adaptation at the Himalayas," Molecular Biology and Evolution, vol. 28, no. 2, pp. 1075-1081, 2011.

[36] F. R. Lorenzo, C. Huff, M. Myllymäki et al., "A genetic mechanism for Tibetan high-altitude adaptation," Nature Genetics, vol. 46, no. 9, pp. 951-956, 2014. 
[37] J. R. Espinoza, G. Alvarez, F. Leon-Velarde et al., "Vascular endothelial growth factor-A is associated with chronic mountain sickness in the Andean population," High Altitude Medicine \& Biology, vol. 15, no. 2, pp. 146-154, 2014.

[38] N. E. Buroker, X. H. Ning, Z. N. Zhou et al., "AKT3, ANGPTL4, eNOS3, and VEGFA associations with high altitude sickness in Han and Tibetan Chinese at the Qinghai-Tibetan plateau," International Journal of Hematology, vol. 96, no. 2, pp. 200213, 2012.

[39] N. E. Buroker, X. H. Ning, Z. N. Zhou et al., "EPAS1 and EGLN1 associations with high altitude sickness in Han and Tibetan Chinese at the Qinghai-Tibetan Plateau," Blood Cells, Molecules \& Diseases, vol. 49, no. 2, pp. 67-73, 2012.

[40] T. Shiina, H. Inoko, and J. K. Kulski, "An update of the HLA genomic region, locus information and disease associations: 2004," Tissue Antigens, vol. 64, no. 6, pp. 631-649, 2004.

[41] M. D. Rossman, B. Thompson, M. Frederick et al., “_HLADRB $1 * 1101_{-}$: A Significant Risk Factor for Sarcoidosis in Blacks and Whites," American Journal of Human Genetics, vol. 73, no. 4, pp. 720-735, 2003.

[42] S. J. Scharf, A. Freidmann, L. Steinman, C. Brautbar, and H. A. Erlich, "Specific HLA-DQB and HLA-DRB1 alleles confer susceptibility to pemphigus vulgaris," Proceedings of the National Academy of Sciences of the United States of America, vol. 86, no. 16, pp. 6215-6219, 1989.

[43] A. Ruiz-de-Leon, J. Mendoza, C. Sevilla-Mantilla et al., "Myenteric antiplexus antibodies and class II HLA in achalasia," Digestive Diseases and Sciences, vol. 47, no. 1, pp. 15-19, 2002.

[44] F. Megiorni and A. Pizzuti, "HLA-DQA1 and HLA-DQB1 in celiac disease predisposition: practical implications of the HLA molecular typing," Journal of Biomedical Science, vol. 19, no. 1, p. 88, 2012.

[45] F. Tekola-Ayele, A. Adeyemo, G. Chen et al., "Novel genomic signals of recent selection in an Ethiopian population," European journal of human genetics : EJHG, vol. 23, no. 8, pp. 1085-1092, 2015.

[46] M. Hanaoka, K. Kubo, Y. Yamazaki et al., "Association of high-altitude pulmonary edema with the major histocompatibility complex," Circulation, vol. 97, no. 12, pp. 1124-1128, 1998.

[47] M. Mazzali, J. A. Jefferson, Z. Ni, N. D. Vaziri, and R. J. Johnson, "Microvascular and tubulointerstitial injury associated with chronic hypoxia- induced hypertension," Kidney International, vol. 63, no. 6, pp. 2088-2093, 2003.

[48] M. Rahman, J. Wang, T. Patterson et al., "Expression of genes related to oxidative stress in the mouse brain after exposure to silver-25 nanoparticles," Toxicology Letters, vol. 187, no. 1, pp. 15-21, 2009. 


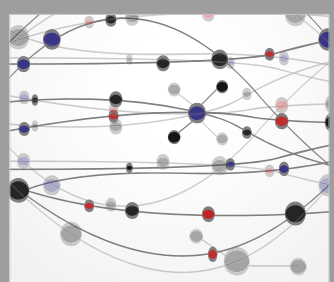

The Scientific World Journal
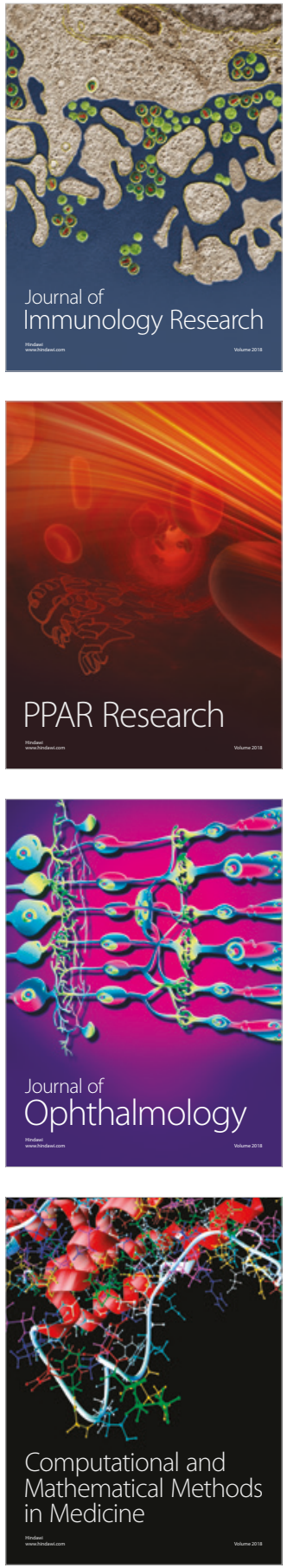

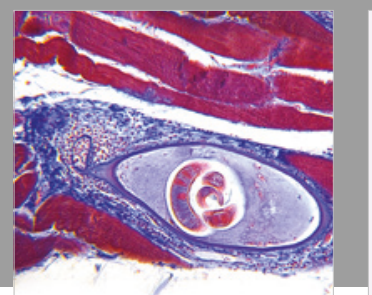

Gastroenterology Research and Practice

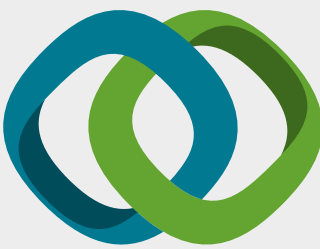

\section{Hindawi}

Submit your manuscripts at

www.hindawi.com
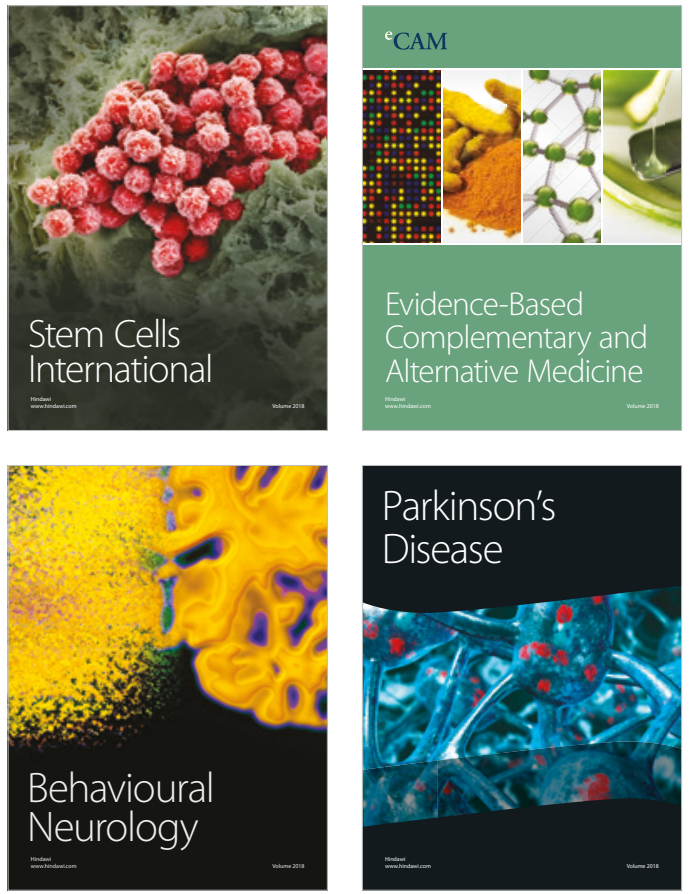

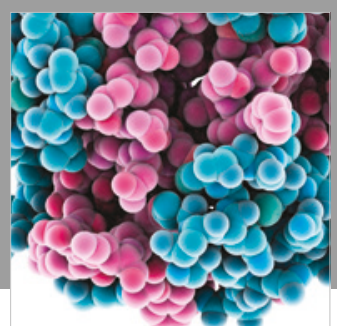

ournal of

Diabetes Research

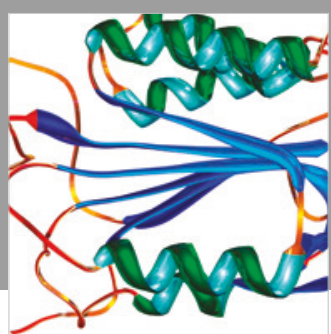

Disease Markers
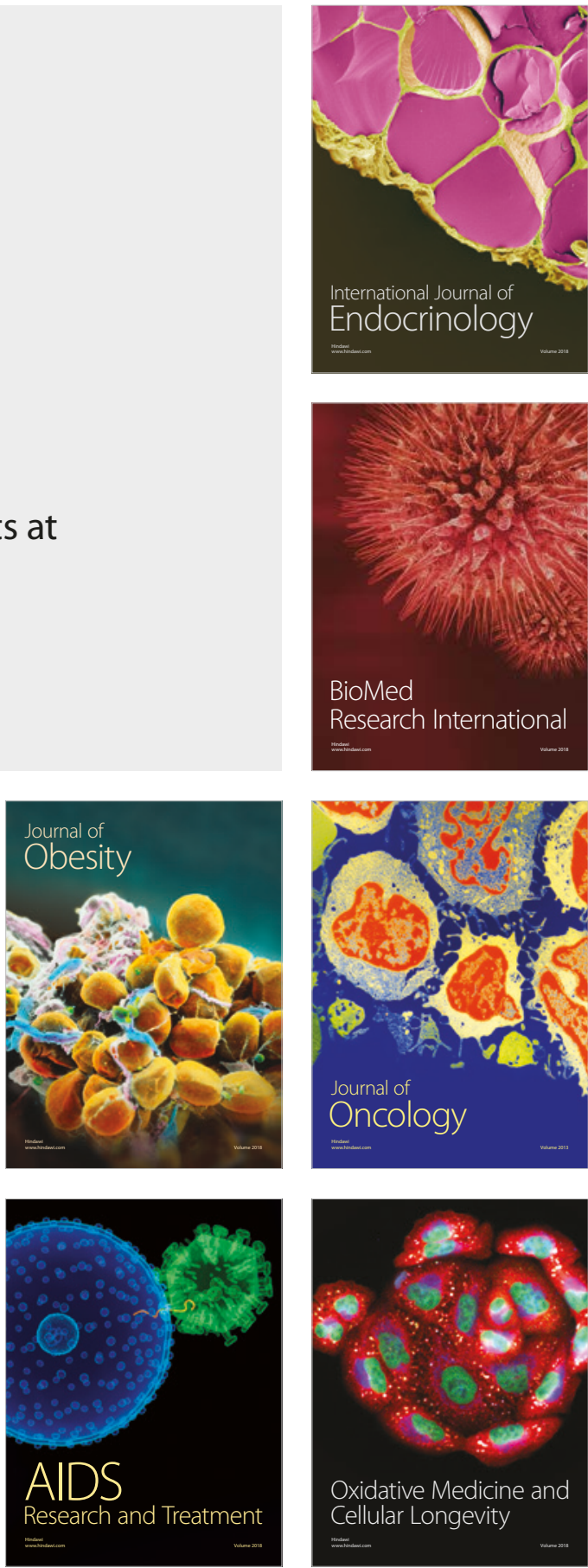